\title{
Stress Fractures of Pars Interarticularis in Adolescent Athletes a Classification System with MRI and CT Enabling Evaluation of The Healing Process
}

\author{
Sundell $\mathrm{CG}^{{ }^{*}}$, Jonsson $\mathrm{H}^{2}$, Ådin $\mathrm{L}^{3}$ and Larsén $\mathrm{K}^{1,4}$ \\ ${ }^{1}$ Department of Community Medicine and Rehabilitation, Sports Medicine Unit, Umeå University, Umeå, Sweden \\ ${ }^{2}$ Department of Orthopaedics, Spinal Unit, Akademiska sjukhuset, Uppsala, Sweden \\ ${ }^{3}$ Diagnostic Radiology, Skellefteå, Sweden \\ ${ }^{4}$ The Swedish School of Sport and Health sciences, Dean's office, Stockholm, Sweden
}

Received: February 24, 2018; Accepted: March 14, 2018; Published: March 23, 2018

*Corresponding author: Claes Göran Sundell, Department of Community Medicine and Rehabilitation, Sports Medicine Unit, Umea University, 90185 Umeå, Tel: +46706870775;E-mail: claes.g.s@telia.com

\begin{abstract}
Introduction: The purpose of this study was to evaluate healing frequency in different stages of stress reactions in the Pars Interarticularis (PI) using a classification system with MRI and CT. The intervention was 3-month rest from physical activity, without a brace, with the exception of activities of daily living.

Materials \& Method: Twelve adolescent athletes with different stages of Spondolysis were included in the study. They had pathology in the Pars Interarticularis and were clinically examined with MRI and CT 3-6 weeks after debut of Low Back Pain (LBP) and re-evaluated after 3 months intervention with rest from physical activity.

Results: A combination of MRI and CT scanning to investigate suspected injuries to Pars Interarticularis in adolescent athletes revealed 6 different stages of Spondolysis that ranged from marrow oedema to pseudoarthrosis.

After 3 months of rest from physical activity the early stages of Pars Interarticularis injuries healed significantly better than the later stages with rest from physical activity.

Conclusion: The combination of MRI and CT revealed 6 stages of stress reactions instead of 4 as in Hollenberg's staging with MRI only. In the 3 earliest stages, of these 6 , rest from physical activity for 3 months can heal the stress reaction.

Keywords: Low Back Pain; Oedema; Pseudoarthrosis; Spondylolysis
\end{abstract}

\section{Introduction}

Adolescent athletes with LBP for more than 3 weeks have radiologically identified pathology of the spinal column in up to about $90 \%$ of the cases [1-3]. More than $48 \%$ of the individuals have stress reactions or fatigue fractures in different stages of healing or pseudoarthrosis in the PI [2-4]. Hollenberg et al. observed in a retrospective study of young athlete's different grades of changes in PI on MRI. He suggested a four-grade classification, early to late changes (Table 1) [5]. Nevertheless, there is a limit to MRI in detecting the exact type of fatigue fracture of the PI. A combination of MRI and CT has been suggested to give a more exact diagnosis of the different stages of PI injuries $[3,6]$.

The early detection and thereby a possibility to heal the fatigue fractures is of importance to avoid secondary complications, mainly spondylolisthesis later in life [7-9].It is also important to know how far the Spondylolysis has progressed as the treatment might be different between the stages.

Table 1: Hollenberg classification of Pars Interarticularis (PI)Abnormalities

\begin{tabular}{|c|c|c|c|c|c|}
\hline & Grade 0 & Grade 1 & Grade 2 & Grade 3 & Grade 4 \\
\hline MRI & No abnormalities in PI & Marrow oedema in PI & $\begin{array}{c}\text { Thinning Fragmentation } \\
\text { Irregularities In PI }\end{array}$ & Complete spondylolysis & Un-united fractures of PI \\
\hline
\end{tabular}

Different interventions have been tried, both surgical and conservative methods. In the conservative treatment, rest is the prime goal of intervention, reached with either prescription of rest or rest and brace but there seem to be difficulties in reaching consensus on the best treatment [1,10-15]. Rest from aggravating physical activity and rest/treatment with a brace have almost the 
same clinical outcome [16].There are several outcome parameters such as clinical healing that means pain relief, measured with Visual Analogue Scale (VAS) and structural healing identified by different imaging methods(X-ray, Spect, CT, MRI) $[17,18]$. Previous studies have shown that rest in combination with orthotics in different forms leads to clinical healing $[1,10]$. It is not known, however, whether the different stages need different treatments to reach structural healing.

Rest from sport activities, with or without a brace, is the treatment mostly recommended [1,19]. In the present study, we thus want to investigate if an intervention with absence of physical activity and sports but without using a brace and with no restrictions in Activities of Daily Living (ADL) supervised by a physiotherapist, will lead to healing and if there are any differences depending on the stage of the stress reaction to the PI.

\section{Materials and methods}

The subjects included in this study is a subgroup from an earlier study. In that study, the inclusion criteria were: adolescents 13-20 years, $\geq 6 \mathrm{~h}$ of sports participation/week and $>3$ weeks of LBP, hindering their ADL and physical activity. The exclusion criteria were: condition that could affect the results and metal plates in the body that could disturb the MRI investigation. At inclusion, prior to treatment, they were evaluated with a questionnaire, physical examination, MRI and CT. A subgroup of fourteen adolescent athletes ( 6 football players, 3 icehockey players, 1 floorball player, 1 basketball player, 1 volleyball player, 1 swimmer and 1 track and field athlete)was diagnosed with stress reactions (using CT and MRI) in the PI ranging from just oedema without fractures to pseudoarthrosis (Spondylolysis) [3]. These individuals were invited to participate in this study. One of them was not interested in further participation in the study. One had a pseudoarthrosis in the L5 level on both sides and thus no healing potential [3]. and was excluded to avoid unnecessary radiation. The remaining twelve patients gave their informed consent to participate in the study. They were recommended refraining from physical activity and sports but not from Activities of Daily Living (ADL) for 3 months, without a brace, and met a physiotherapist once a month to ensure adherence/compliance. At each of these meetings the perception of pain was registered with Visual Analog Scale (VAS) [20]After three months of intervention, a new MRI and CT were performed.

\section{MRI and CT examinations}

The MRI was performed with a different protocol compared to the standard for adults.

The protocol focused on sequences sensitive to bone marrow and soft tissue oedema to detect the secondary bone marrow changes that come with a fatigue fracture. A sagittal T1- weighted FSE sequence, sagittal T2-weighted fat suppressed FSE sequence and a coronal T2-weighted inversion recovery (STIR) sequence were used.

The initial CT was performed in all cases on the L4 and L5 vertebrae level regardless of MRI findings. If there were any findings suspicious to fatigue fracture in levels superior to L4 on the MRI, the intention was to include this level on the CT. The CT control after three months of rest was only performed on the level of pathology. Consecutive $0.6 \mathrm{~mm}$ thick slices with a bone algorithm were used and evaluated on a workstation. A radiologist with long experience in MRI and CT diagnostic imaging read the MRI and CT results. MRI was performed on a standard 1.5 T Philips Achieve systems and CT was performed on a General Electric Light Speed VCT 64 row detector system. The MRI examination was evaluated in search of changes compatible with a fatigue fracture, which was the presence of high signal in the bone marrow in the PI of the vertebrae on T2 weighted fat suppressed sequences and low signal on $\mathrm{T} 1$ weighted sequences. The MRI and CT investigations were performed after $11-20$ weeks following the start of the intervention (median 14 weeks).

The Hollenberg classification on MRI was as follows:

$\rightarrow 0=$ no signal abnormalities,

$\rightarrow 1=\mathrm{T} 2$ signal abnormalities in PI - marrow oedema,

$\rightarrow 2$ = $\mathrm{T} 2$ signal abnormalities - thinning, fragmentation or irregularity of PI,

$\rightarrow 3=\mathrm{T} 2$ signal abnormalities - visible complete unilateral or bilateral Spondylolysis,

$\rightarrow 4$ = No abnormal T2 signal - complete Spondylolysis not united fracture of PI was our basic classification, but we added CT in our investigation to get a more specified diagnosis.

\section{Statistics}

Non-parametric Related Samples Wilcox on Sign Rank Test was used in our estimates of $p$ value for changes in the perception of pain. To analyse differences in healing among the different stages X2 test with Yates correction method was used due to the small number of cases.

\section{Results}

Forty-Eight PIs were evaluated in the twelve athletes. In our classification, according to a modified Hollenberg, we added a CT investigation to give a more precise diagnose of the PIs affected. This further classification was also used at the re-examination after the three-month period of rest.

The classification modified by using the combination of MRI and CT supports the following stages (Figure 1):

$\rightarrow 0$ - no oedema on MRI, normal CT,

$\rightarrow 1$ - oedema in PI on MRI, no sign of fracture on CT,

$\rightarrow 2$ - oedema in PI on MRI, incomplete fracture in PI on CT,

$\rightarrow 3$ - oedema in PI on MRI, complete fracture of PI on CT,

$\rightarrow 4$ - oedema in PI on MRI, signs of healing of PI on CT (periosteal callus, sclerosis, reduced extent or gap of fracture)

$\rightarrow 5$ - no oedema in PI on MRI, healed fracture in PI on CT

$\rightarrow 6$ - no oedema in PI on MRI, Pseudoarthrosis in PI on CT.

When including the CT examination to the MRI investigation we 


\section{MR/CT Classification System for Lumbar PI}

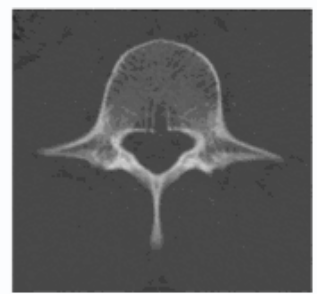

No oedema on MRI-normal CT(stage 0)

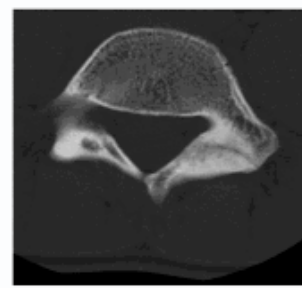

oedema on MRI-incomplete

fracture on $\mathrm{CT}$ (stage 2)

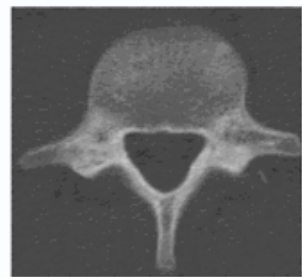

oedema on MRI-signs of

healing on CT(stage 4)

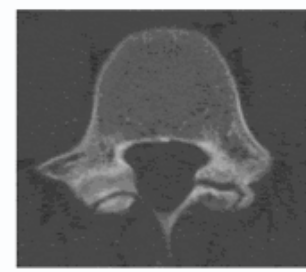

No oedema on MRI-

pseudoarthrosis on $\mathrm{CT}$ (stage 6)

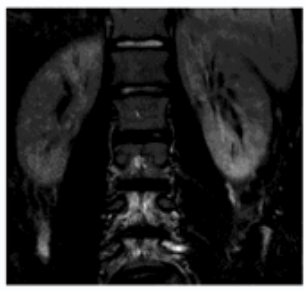

S TIR(short tau-inversion recovery sequence)-o€ in $\mathrm{PI}$ on MRI(stage 1)

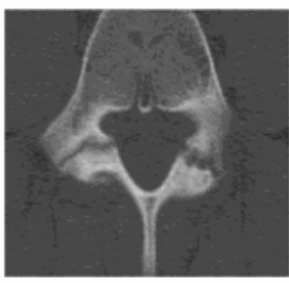

oedema on MRI-complete

fracture on $\mathrm{CT}$ (stage 3 )

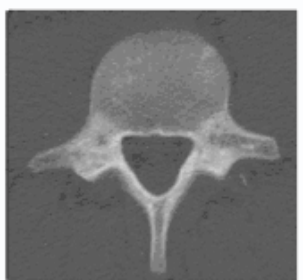

No oedema on MRI-

healed fracture on $\mathrm{CT}$ (stage 5)

Figure 1: MR/CT Classification System for Lumbar PI

found: four PIs with oedema, six PIs with incomplete fractures, three PIs with complete fracture, 3 PIs with signs of healing and four PIs with pseudoarthrosis (Figure 2) were found, already reported [3].

At the examination after the intervention there were no new developed stress reactions. Five PIs developed pseudoarthrosis (one PI with only oedema on the first MRI/CT investigation, two
PIs with incomplete fracture, two PIs with complete fracture). Three out of the four PIs with only oedema and no visible fracture on first CT, healed, 4 out of six PI with incomplete fracture were healed, 1 out of 3 with complete fracture healed and 3 out of 3 with early signs of healing, healed. None of the 7 PIs with radiological signs of pseudoarthrosis had healed or showed signs of healing after intervention. In total 11 out of 16 PIs healed after the three months (Figure 2). If the PIs were divided into 


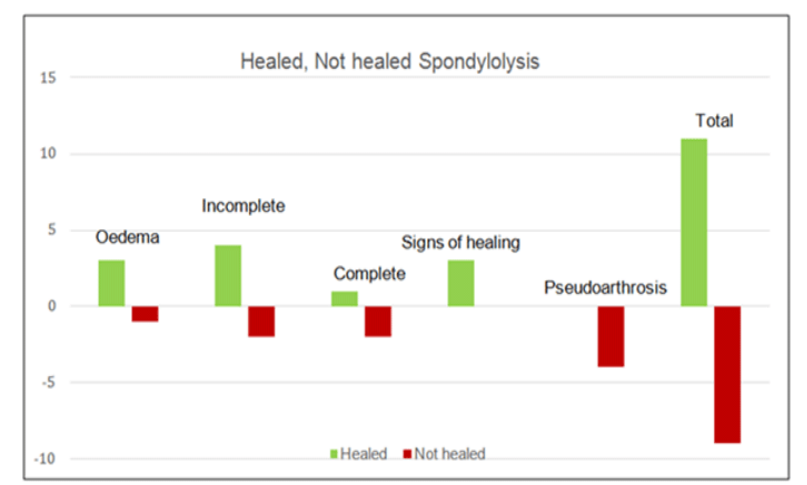

Figure 2: MRI/CT after recommended absence from physical activity and sports but no restrictions in Activities of Daily Living (ADL) for 3 months in 12 individuals. Four PIs per individual adds up to 48 investigated PIs. Normal PI was found in 28 PIs, and 20 PIs displayed different stages of stress reactions to the PI at inclusion. Out of these 20 PIs 11 healed PIs were found and 5 PIs had developed Spondylolysis/ Pseudoarthrosis at follow up after 3 months. Early conditions (Grade 1, $2,4)$ heals significantly better than the later conditions (Grade 3,6$)(p$ $<0.01)$. Grade 5 is not included in the figure since it was healed already at inclusion

early stage (oedema, incomplete fracture and signs of healing) and late stage (complete fracture and pseudoarthrosis) we found a significant difference in healing frequency after three months of intervention where more of the early stages healed compared to the later stages. The early signs of injury (oedema, incomplete fracture with or without signs of healing) thus, seem to heal better than PIs with signs of complete fracture and pseudoarthrosis[11/13(84,6 \%)versus 1/7(14\%)] $(\mathrm{p}<0.01)$.

One athlete with complete fracture developed pseudoarthrosis, and had to be operated, as she had no relief of Low Back Pain (LBP) after three months recommended intervention and rehabilitation for 2 months. One athlete with oedema and an incomplete fracture healed but nonetheless had no relief of pain. A third athlete had a progression from an oedema on one side and an incomplete fracture on the other side of the same spinal level between the first and second MRI and CT investigations and developed pseudoarthrosis on both PIs after intervention.
At baseline, we measured LBP when it was at its worst with VAS scale (0-100 $\mathrm{mm})$. The same measurements were performed after the three months of intervention.

The perception of pain registered on VAS decreased between baseline and after the end of intervention (72.4 and 27.3, $\mathrm{p}=0.004$ ) indicating a large decrease in pain during the three months' period of changes in activity level. If the cases were divided into completely healed PIs and not completely healed PIs, no difference in decrease of VAS was found, between baseline and after three months' rest from vigorous physical activity. Healed PI decreased from 67.6 to 25.4 whereas not healed PI decreased from 73.3 to 33.0. (n.s., p <0,485)thus pain relief is not a good measurement on healing.

\section{Discussion}

Hollenberg et al. created a classification system for the injury to PI based on MRI investigation and the hypothesis that Spondylolysis develops in stages due to repetitive trauma [5]. In their retrospective study of a group of young athletes with low back pain they found different stages of stress-related marrow oedema in PI in $42 \%$ of their population and many with unilateral reactions. We also observed unilateral as well as variable changes in the PI in young athletes [3].The development of CT and MRI have made it possible (compared to X-ray and Scintigrafi) to use the combination MRI and CT to make more precise judgments in this process (Table 2). After three months of intervention, in the present study, MRI and CT displayed higher frequency healing in PI with early stages than in late stages. The early signs of injury (oedema, incomplete fracture with or without signs of healing) seem to heal better (eleven out of thirteen, 84,6\%) than PIs with signs of complete fracture and pseudoarthrosis ( 1 out of 7 healed, $14 \%)$ with rest $(\mathrm{p}<0.01)$. This would support the hypothesis of stage development Spondylolysis [5].

Adding CT to MRI examination helped to separate stage 2-4 more precisely into incomplete, complete fracture and signs of healing. If all PIs with oedema were not further diagnosed, we would not have identified the difference in healing between oedema with or without a fracture. The four PIs without oedema that had pseudoarthrosis would not have been diagnosed.

About $15 \%$ of the individuals with Spondylolysis develop

Table 2. Modified Hollenberg classification

\begin{tabular}{|c|c|c|c|c|c|c|c|}
\hline & Grade 0 & Grade 1 & Grade 2 & Grade 3 & Grade 4 & Grade 5 & Grade 6 \\
\hline & $\begin{array}{c}\text { No oedema } \\
\text { in PI on MRI, } \\
\text { normal CT }\end{array}$ & $\begin{array}{l}\text { Oedema in } \\
\text { PI on MRI, } \\
\text { no sign of } \\
\text { fracture on CT }\end{array}$ & $\begin{array}{l}\text { Oedema in } \\
\text { PI on MRI, } \\
\text { incomplete } \\
\text { fracture in PI } \\
\text { on CT }\end{array}$ & $\begin{array}{l}\text { Oedema in PI on } \\
\text { MRI, complete } \\
\text { fracture in PI } \\
\text { on CT }\end{array}$ & $\begin{array}{l}\text { Oedema in PI on MRI, } \\
\text { signs of healing in } \\
\text { PI on CT (periosteal } \\
\text { callus, sclerosis, } \\
\text { reduced extent or gap of } \\
\text { fracture) }\end{array}$ & $\begin{array}{l}\text { no oedema } \\
\text { in PI on } \\
\text { MRI, healed } \\
\text { fracture in PI } \\
\text { on CT }\end{array}$ & $\begin{array}{c}\text { No oedema } \\
\text { in PI on MRI, } \\
\text { Pseudoarthrosis in } \\
\text { PI on CT }\end{array}$ \\
\hline CT & No Sign & No Sign & Sign & Sign & Sign & Sign & Sign \\
\hline MR & No Sign & Oedema & Oedema & Oedema & Oedema & No Sign & No Sign \\
\hline
\end{tabular}


spondylolisthesis [10].Decades after the initial lesion a combination of disc degeneration and slip at the Spondylolysis level can become symptomatic [9]. Sonne-Holm et al. found in a cross-sectional study of a general population (aged 22-93 years) that Lumbar Spondylolysis prevalence increases during life [21]. Several authors report that a manifest pseudoarthrosis does not heal $[1,3,10,13,22]$. According to the present study early intervention has significantly better effect on the healing process. Thus, early detection, examination and radiological evaluation, of young athletes with LBP can be of great importance for the healing process.

Different interventions have been tried, both surgical and conservative methods. In the conservative treatment, rest is the prime goal of intervention, reached with either prescription of rest or prescription of rest and brace [1,10-14,23]. Rest and rest/brace have almost the same clinical outcome [16]. This was the reason why we choose only to rest from physical activity as treatment.

Structural healing is not the same as clinical recovery (pain relief). In this study, there was no significant difference in pain relief (VAS) between patients with and without healed PI. The study focused on the healing process within three months and the observation time is too short to evaluate clinical outcome.

Structural healing might be necessary to avoid disc degeneration and secondary spondylolisthesis later in life $[9,21]$. It remains to be shown that early healed PI remains healed in the long-term.

This study shows that rest from sports and physical activity alone lead to a high degree of healing in the early stages of injury to the PI (Figure 2). Early recognition and intervention will thus prevent the need of a brace, a great impact in a young person's life.

Later stage of injury identified with CT might need a brace to heal properly $[1,14,23]$ As we may identify, the complete fracture, with the combination MRI and CT where an oedema on MRI is used as a guide to the level/levels that need a CT for specific stage diagnosis (to identify stage 3 ) the radiation is kept at a level of 0.5 - $1.5 \mathrm{mSv}$ for each level (compared to approx. $1.8 \mathrm{mSv}$ on a usual $\mathrm{X}$-ray investigation)

In conclusion, we found that Early identification of injuries to PI in young athletes with LBP is of importance, since more healing is seen if rest from physical activity and sport is recommended early in the process. Early investigations with MRI and CT thus are of great importance in young athletes with LBP.

A weakness of the study is the fact that there are few participants in the study, so these results must be confirmed in other similar studies. The follow-up time is too short to evaluate if the healing of the PI has any influence on the clinical outcome and if the PI remains healed in the long-term.

\section{Conclusion}

Early identification of injuries to PI in young athletes with LBP should be important, since more healing is seen if rest from physical activity and sport is recommended early in the process. Early investigations with MRI and CT are of great importance in young athletes with LBP.

- A new classification for staging of Spondylolysis with a combination of MRI and CT

- Early stages of reactions in PI in the lower back seem to heal better than late stages

- Absence of physical activity and sports but no restrictions in activities of daily living for 3 months without a brace can heal early stages of stress reactions

\section{Acknowledgements}

This study was supported by Swedish National Centre for Research in Sports (CIF).

\section{Declarations \\ Conflict of interest}

We declare that we have no conflicts of interest in the authorship or publication of this contribution.

\section{Ethical Approval}

The investigation was approved by The Regional Ethics Committee at Umeå University (06-014)

\section{Author's contribution}

$\mathrm{KL}$ is professor and head tutor; $\mathrm{HJ}$ is assistant tutor and orthopaedic surgeon; LA is radiologist and CGS is the corresponding author. All authors, contributed substantially to the writing of this paper, have read the paper and approved the final version of the manuscript.

\section{References}

1. Fujii K, Katoh S, Sairyo K, Ikata T, Yasui N. Union of defects in the pars Interarticularis of the lumbar spine in children and adolescents. The radiological outcome after conservative treatment. J Bone Joint Surg. 2004;86(2):225-231. Doi:10.1302/0301-620X.86B2

2. Kujala U M, Kinnunen J, Helenius P, Orava S, Taavitsainen, M, Karaharju E. Prolonged low-back pain in young athletes: a prospective case series study of findings and prognosis. Eur Spine J. 1999;8(6):480-484.

3. Sundell CG, Jonsson H, Ådin L, Larsen K. Clinical examination, spondylolysis and adolescent athletes. Int J Sports Med. 2013;34(3):263-267. Doi:10.1055/s-032-1321723

4. Micheli LJ, Wood R. Back pain in young athletes. Significant differences from adults in cause and patterns. Arch Pediatr Adolesc Med. 1995;149(1):15-18.

5. Hollenberg GM, Beattie PF, Meyers SP, Weinberg EP, Adams MJ. Stress reactions of the lumbar pars interarticularis. The Development of a new MRI classification system. SPINE.2002;27(2):181-186.

6. Kobayashi A, Kobayashi T, Kato K, Higuchi H, Takagishi K. Diagnosis of radio graphically occult lumbar spondylolysis in young athletes by Magnetic Resonance Imaging. The American journal of Sports Medicine.2013;41(1):169-176. Doi: 10.1177/0363546512464946 
7. Fredrickson BE, Baker D, McHolick WJ, Yuan HA, Lubicky JP. The natural history of Spondylolysis, and spondylolisthesis in children and adolescents. J Bone Joint Surg Am. 1984;66(5):699-707.

8. Morita T, Ikata T, Katoh S, Miyake R. Lumbar spondylolysis in children and adolescence. J Bone Joint Surg. 1995;77(4):620- 625.

9. Floman Y. Progression of lumbosacral isthmic spondylolisthesis in adults. Spine. 2000;25(3):342-347.

10. Hu SS, Tribus CB, Diab M, Ghanayem AJ. Spondylolisthesis and spondylolysis, Bone Joint Surg Am. 2008;90 (3):656-671.

11.Jackson DW, Wiltse LL, Dingeman RD, Hays M. Stress reactions involving the pars interarticularis in young athletes. Am J Sports Med.1981;9(5):304-312. Doi: 10.1177/036354658100900504

12. Ruiz-Cotorro A, Balius-Matas R, Estruch-Massana A, Vilaro Angulo J. Spondylolysis in young tennis players.Br J Sports Med. 2006;40:441446. Doi:10.1136/bjsm.2005.023960

13. Standaert CJ, Herring SA. Expert opinion and controversies in sports and musculoskeletal medicine: The Diagnosis and Treatment of Spondylolysis in Adolescent Athletes. Arch Phys Med Rehabil. 2007;88(4):537-540. Doi: 10.1016/j.apmr.2007-01.00

14. Sys J, Michielsen J, Bracke P, Martens M, Verstreken J. Non operative treatment of active Spondylolysis in elite athletes with normal x-ray findings: literature review and results of conservative treatment. Eur Spine J. 2001;10(6):498-504. Doi:10.1007/s005860100326

15. Garet M, Reiman M, Mathers J, Sylvain J. Nonoperative treatment in lumbar spondylolysis and spondylolisthesis: A systematic review. Sport Health. 2013;5(3):225-232. Doi: 10.1177/1941738113480936

16. Alvares-Diaz P, Alentorn-Geli E, Steinbacher G, Rius M, Pellisé F, Cugat R. Conservative treatment of lumbar spondylolysis in young soccer players. Knee Surg Sports Traumatol Arthrose. 2011;19(12):21112114. Doi:10.1007/s00167-011-1447-2114
17. Trout AT, Sharp SE, Anton CG, Mehlman CT. Spodylolysis and Beyond: Value of SPECT/CT in Evaluation of Low Back Pain in Children and Young Adults. Radio Graphics. 2015;35(3):819-834. Doi: 10.1148/ rg.2015140092

18. Gregory PL, Batt ME, Kerslake RW, Scammell BE, Webb JF. The value of combining single photon emission computerised tomography in the investigation of Spondylolysis. Eur Spine. J. 2004;13(6):503-509. Doi: 10.1007/s00586-004-0696-2

19. Leone A, Cianfoni A, Cerase A, Magarelli N, Bonomo L. Lumbar spondylolysis - a review. Skeletal Radiol. 2011;40(6):683-700. Doi: 10.1007/s00256-010-0942-0

20.Sugiura S, Aoki Y,Toyooka T, Shiga T, Otsuki K, Aikawa E, et al. Characteristics of Low Back Pain in Adolescent. Patients with EarlyStage Spondylolysis Evaluated Using a Detailed Visual Analogue Scale. SPINE. 2014;40(1): E29-E34. Doi:10.1097/BRS.0000000000000657

21. Sonne-Holm S, Jacobsen S, Rovsing HC, Monrad H, Gebuhr P. Lumbar spondylolysis: a lifelong dynamic condition? A cross sectional survey of 4.151 adults. Eur Spine J. 2007;16:821-828. Doi: 10.1007/s00586006-0250-5

22. Motley G, Nyland J, Jacobs J, Caborn D. The pars interarticularis stress reaction, spondylolysis, and spondylolisthesis progression. Journal of Athletic Training. 1998;33(4):351-358.

23. Sairyo K, Sakai T, Yasui N, Dezawa A. Conservative treatment for pediatric lumbar spondylolysis to achieve bone healing using a hard brace: what type and how long. J Neurosurg Spine.2012;16(6):610-614. Doi: 10.3171/2012.2. SPINE10914 